\title{
Application of the PCA to Guided Ultrasonic Waves to Evaluate Tensile Stress in a Solid Rod
}

\author{
JABID QUIROGA MÉNDEZ, RODOLFO VILLAMIZAR MEJÍA, \\ JOHN L. QUIROGA PINEDA, LUIS E. MUJICA \\ and MAGDA RUIZ
}

\begin{abstract}
In this work, the use of Principal Component Analysis (PCA), through $\mathrm{T}^{2}$ and Q-statistics, is proposed to evaluate the tensile stress in a bar rod by means of ultrasonic guided waves. This method can be potentially used in-situ and real time for continuous condition monitoring. The specimen tested is a 1020 steel bar of 1" cold finished with yield strength of $441 \mathrm{MPa}$. Piezoelectric transducers are used in a through-transmission configuration to excite and detect axis-symmetric waves at the free ends of the bar. The load on the bar is performed using a MTS $^{\circledR}$ testing machine in load control to maximum load of $80 \%$ of the yield strength. A dedicated Matlab $^{\circledR}$ software is utilized to perform ultrasonic signal generation, signal acquisition and processing. A 5 cycles Gaussian-modulated sinusoidal pulse is used to generate narrow-band waves. Data captured by the piezoelectric sensor for each load condition are projected into the PCA model. The first two projections, $Q$ statistic and $T^{2}$-statistic indices are analyzed. Results of each load scenario are presented and discussed demonstrating the feasibility and potential of using this formulation in the evaluation of the tensile stress in structural elements.
\end{abstract}

\section{INTRODUCTION}

The importance of low frequency ultrasonic guided waves in the field of nondestructive testing and structural health monitoring has increased considerably in recent years. The ultrasonic guided waves have the ability to be propagated along relative long distances, compared to most bulk wave NDE methods, while still maintaining sensitivity to condition changes in the structure. Thus, guided waves are used for damage detection, damage localization and also for material characterization.

Jabid Quiroga ${ }^{2}$, Rodolfo Villamizar ${ }^{2}$, John Quiroga ${ }^{2}$, Luis Mujica ${ }^{1}$, Magda Ruiz ${ }^{1}$ \{magda.ruiz, luis.eduardo.mujica\}@upc.edu. Escola Universitària d'Enginyeria Tècnica Industrial de Barcelona. Consorci Escola Industrial de Barcelona. Universitat Politècnica de Catalunya. BARCELONATECH, Departament of Applied Mathematics III.

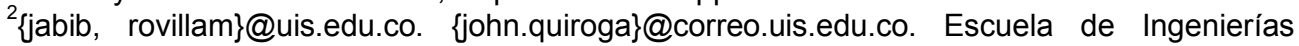
Eléctrica, Electrónica y de Telecomunicaciones (E3T), Universidad Industrial de Santander (UIS). Grupo de Control Electrónica Modelado y Simulación (CEMOS). Santander, Colombia. 
Stress conditions in a structure influence on servicing of engineering components, affecting their strength, expected operational life and dimensional stability. Stress measurement by ultrasonic waves is a reasonably easy and inexpensive method, recently used in some industrial applications. However, it is slightly sensitive to the microstructure effects like grain size [1], texture and structure [2-4] and also to the operating conditions such as temperature [5], coupling [6].

The guided waves propagating under stress conditions present some changes in the wave pattern, which can be monitored under two approaches. The first one is based on the Acoustoelastic Effect, which involves the evaluation of the change of ultrasonic bulk wave velocities. The second one relies on particular features in the waveform attributed to the propagation in a medium under stress. These changes can be traced by using statistics tools, such as PCA, and time and frequency indicators.

The theoretical framework that explains the dependence of wave speed on stress, or acoustoelasticity, was developed by Hughes and Kelly by applying the Murnaghan theory of finite deformation on the propagation of bulk elastic waves in an initially isotropic solid subjected to a stress [7]. They specifically considered uniaxial stress and derived expressions for changes in shear and longitudinal wave velocities as a function of applied stress for known material properties. Hughes and Kelly determined that for isotropic materials subject to uniaxial stress, in addition to the two Lamé constants, $\lambda$ and $\mu$, three additional constants, $l, m, n$ are required to describe the relation between stress and velocity. Once the values of $l, m, n$ of a particular material specimen are determined, any experimental measurement of one of the bulk velocities reveals the stress at which the specimen is subject to.

The main propose of [7] was the measurement of Third Order Elastic Constants (TOEC); however, this focus was largely modified to the measurement of both applied and residual stresses [8]. The TOEC highly depends on the material processing, such as casting, rolling, or drawing. This processing dependence was traced to the presence of residual, or internal, stresses. The level, depth, and location of residual stresses influence the acoustoelastic properties. So, a new stage of applications has started by using acoustoelasticity to measure residual stress [9].

Recent researches on the Acoustoelastic Effect have been oriented to estimate residual stress. Three major difficulties have prevented the advance of this technology [10]. First, the acoustoelastic effect is small for metals, typically about $0.001 \%$ per MPa of applied stress, thus a high precision in experimentation is required. Second, the inherent or induced preferred orientation of crystalline grains affects acoustoelasticity; this orientation, called texture, causes an anisotropic effect in the material properties. The third major difficulty is the unknown influence of localized plastic deformation, which is closely related to residual stresses. Although the model of the acoustoelasticity predicts the change of ultrasonic bulk velocities, some recent works have shown the extension of this behavior to the guided waves.

Therefore, a new subject of research has been gaining the attention, the disperse behavior of guided waves propagating in a medium under stress, where the propagation velocity does not depend only of the frequency but also of the stress level. Some recent works have been using this behavior [11,12], where the longitudinal mode $\mathrm{L}(0,1)$ is used to track the variations of the phase velocity in a rod waveguide expose to stress. In [13] the dispersion curves for a plate are initially obtained consider it isotropic but under uniaxial stress becomes anisotropic. 


\section{PRINCIPAL COMPONENT ANALYSIS}

PCA is a statistical tool with a wide variety of uses: data dimensional reduction, pattern recognition, data compression and extraction, and statistical modelling. The goal of PCA is to discern among a set of data, which are the most significant for the system, the redundant data and the noise. This is achieved by representing the data information on a new space (coordinates) along directions of maximal variance and minimal redundancy, where noise and redundancies are filtered.

SHM based on PCA has been extensively applied for extracting structural damage features and to discriminate features from damaged and undamaged structures [14-16], among others applications. In the above references just the principal components or theirs scores were studied, however, PCA methodology provides additional statistical features that can be considered as indices [17].

For SHM based on PCA the data can be arranged in a matrix $\mathbf{X}$, which contains information from the measurement $j$ and $n$ experimental trials. Each row vector $\left(x_{i}\right)$ represents measurements from the sensors attached to the structure for a specific experiment trial. In the same way, each column vector $\left(\vartheta_{j}\right)$ represents samples from sensors (one variable) in the whole set of experiment trials. Usually, each sensor vector $\vartheta_{j}$ is re-scaled to have zero mean and unity variance.

The covariance matrix $\mathbf{C}_{\mathbf{x}}$ of $\mathbf{X}$ is obtained, where the eigenvectors of $\mathbf{C}_{\mathbf{x}}$ are the column vectors of the transformation matrix $\mathbf{P}$. This matrix forms a basis in the $m$ dimensional space [17], thus the property expressed by (1) must be satisfied:

$$
C_{X} P=P \Lambda,
$$

where, $\Lambda=\operatorname{diag}\left(\lambda_{1}, \lambda_{2}, \ldots \lambda_{m}\right)$. The transformation matrix $\mathbf{P}$ is used to transform the original data matrix $\mathbf{X}$ into the form:

$$
T=X P \text {. }
$$

The eigenvectors $p_{j}$ forming the transformation matrix $\mathbf{P}$ are sorted by descending order, according to the eigenvalues and they are called the Principal Components of the data set. The eigenvector with the highest eigenvalue is the most representative component for the data with the largest quantity of information. Geometrically, the $j^{\text {th }}$-column vector $t_{j}$ of the transformed data matrix $\mathbf{T}$ is the projection of the original data over the direction of vector $p_{j}\left(j^{\text {th }}\right.$ principal component). The projection of the data in the new frame is characterized by being uncorrelated and having the maximal data variance, thus it can be potentially the best representation of the process features.

Since eigenvectors are ordered according to the amount of information, it is possible to reduce the dimensionality of the data set $\mathbf{X}$ by choosing only a reduced number $r$ of principal components, those corresponding to the first eigenvalues.

Two statistics indices associated to the PCA are the Hotteling $T^{2}$ statistics and the $Q$ statistics. The first one is based on analyzing the residual data matrix $\tilde{X}$ to represent the variability of the data projection in the residual subspace. It only detects variations in the subspace of the first $r$ principal components, which are greater than those that can be explained by the common-cause variations. In other 
words, $T^{2}$-statistic is a measurement of the variation of each sample within the PCA model. The $T^{2}$-statistic of the $i^{\text {th }}$ sample (or experiment) is defined in the form of Eq.(3) .

$$
T_{i}^{2}=\sum_{j=1}^{r} \frac{t_{s i j}^{2}}{\lambda_{j}}=t_{s i} \Lambda^{-1} t_{s i}^{T}=x_{i} P \Lambda^{-1} P^{T} x_{i}^{T}
$$

Besides, $Q$-statistic denotes the change of the events that are not explained by the principal components model. It is a measurement of the difference, or residual, between a sample and its projection into the model. The $Q$-statistic of the $i^{\text {th }}$ sample (or experiment) vector $x_{i}$ is defined as follows by Eq. (4):

$$
Q_{i}=\tilde{x}_{i} \tilde{x}_{i}^{T}=x_{i}\left(I-P P^{T}\right) x_{i}^{T},
$$

where, $\tilde{x}_{i}$ is the $x_{i}$ projection into the residual subspace.

\section{PCA BASED STRESS MONITORING APPROACH}

In this work, a piezo-actuated steel rod under stress is studied. Changes in the ultrasonic guided wave propagating along it are discriminated by using PCA statistical indices. Information of the wave in its nominal stage is statistically modeled (baseline model) by means of the principal components obtained from PCA analysis. The diagnosis stage is performed using the $T^{2}$ and $Q$-Statistics indices calculated from the principal components of the experiment under analysis. These components are projected by means of the baseline model.

Also, in the proposed methodology, the actuated and sensed signals are coupled by using cross-correlation presented in Eq. (5), before computing the PCA model, as follows.

$$
x_{i}(k) \stackrel{\text { def }}{=} \sum_{m=-\infty}^{\infty} f^{*}[m] g[m+k] \text {, }
$$

where, $f^{*}$ denotes the complex conjugate of $f$ (actuated signal) and $g$ is the sensed signal. In the proposed PCA model, the cross-correlation is arranged in the matrix $\mathbf{X}$. One experiment consists of one actuated signal and a set of sensed signals ( $I$ signals $x K$ samples per experiment), thus the normalized matrix $\mathbf{X}$ represents the set of cross-correlated signals (actuated and sensed) per sensor. The baseline model (P matrix) is built using a set of experiments (tipically 100) of the steel rod without stress or normal condition.

In the diagnosis phase, several experiments are conducted over the steel rod in order to determine its stress states. A new matrix $\mathbf{X}$ is arranged with the captured data, where the number of collected samples (data-points) must be the same that the used in the modelling phase. This matrix is projected into the PCA model using Eq. (6). Projections onto some of the first components are obtained and the stress indices $\left(T^{2}\right.$-statistic and $Q$-statistic) are calculated and compared with the baseline values. The general procedure for detecting and distinguishing stresses on structures based on PCA can be summarized (see Figure 1). 


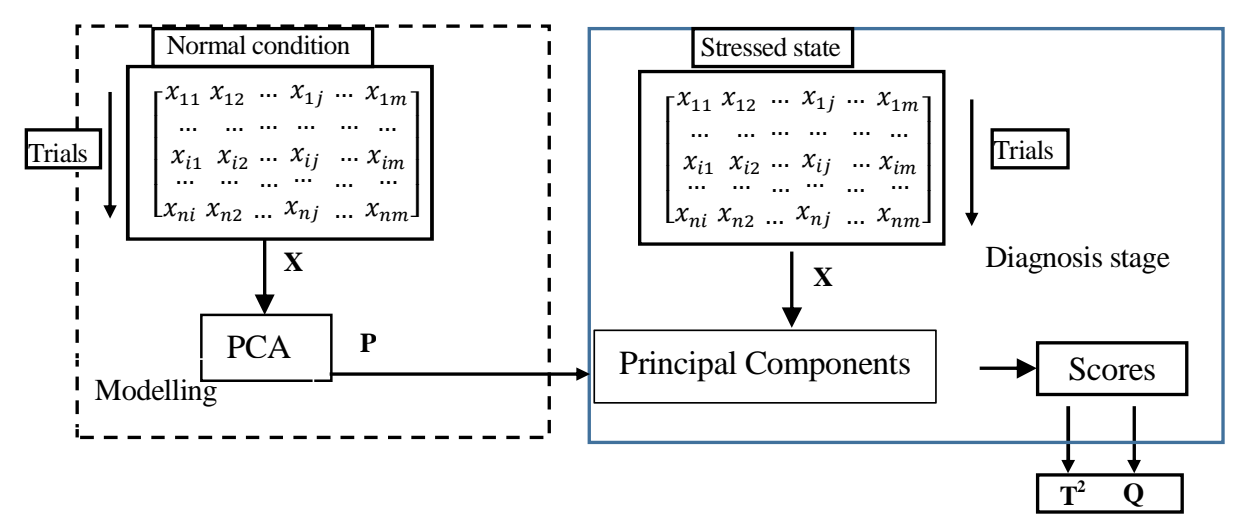

Figure 1: General scheme based on PCA for detecting and distinguishing stress in structures.

\section{PCA INDICES SENSIBILITY}

According to acoustoelasticity theory and previous experimental results, the ultrasonic wave velocities change under variations in the stress level of the medium. This behavior has also seen by the authors in previous experiments (see Figure 2)

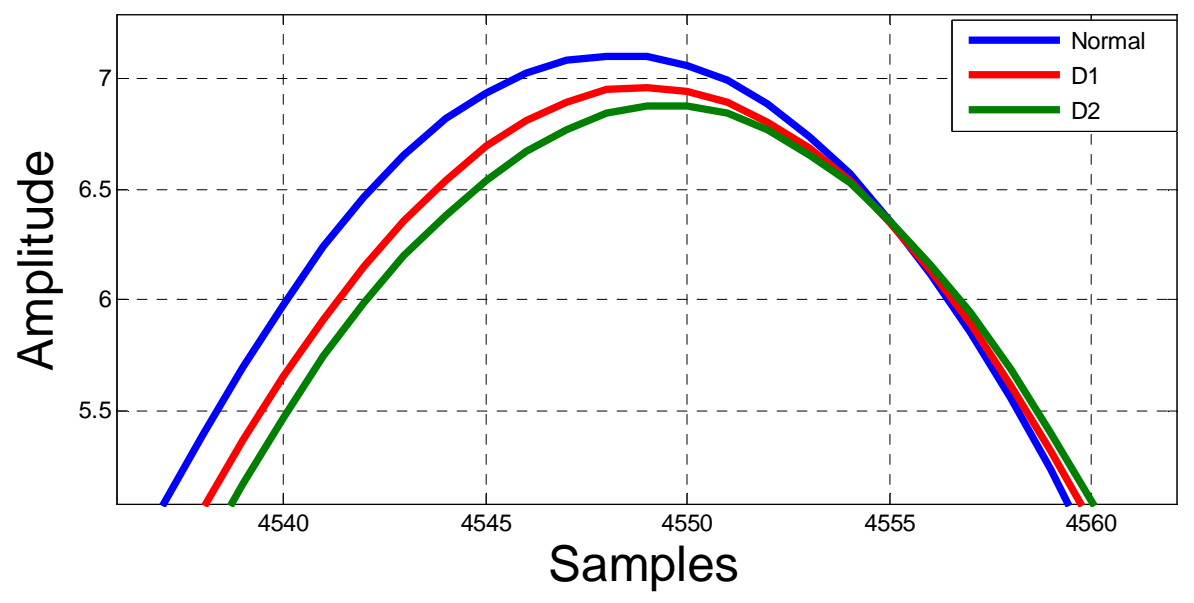

Figure 2: Experimental results of guided waves under different stress scenarios

In this work, PCA has been used to detect changes in the guided wave with respect to a reference wave (baseline). It can be observed changes in amplitude and phase velocity in waves for each one of the different stressed scenarios studied (see Figure 2). Therefore, a study of sensibility of the indices $T^{2}$ and $Q$-Statistics in presence of these variations must be carried out.

Simulated packets of guided waves are used to replicate the increase (steps of gain of $1 \%$ ), the attenuation (steps of $1 \%$ of attenuation), and the deviations on velocity (steps of $0.4 \%$ of change of the nominal velocity) with respect to the original wave to trace differences in the values of $T^{2}$ and $Q$-Statistics.

According to figures 3-4, it can be noted a linear relationship between the indices $Q$ and $T^{2}$ for variations in amplitude and velocity of the guided wave. Thus, only the $Q$-Statistics index could be chosen as a sensibility factor $S F$, see eq (6), to study its variation when scaled and delayed versions of the guided waves are projected on the principal components. 


$$
S F=\frac{\Delta Q}{\Delta V}
$$

where, $\Delta V$ is change in amplitude or velocity.

Similar amplitude change percentage, either attenuation or amplification, produces the same coordinates in the diagram $T^{2}-Q$ (see Figure 3 ). Thus, the indices cannot distinguish if the variation is amplification or attenuation.

Figure 4 presents different coordinates of $Q$ and $T^{2}$ for each increasing and decreasing velocity of the nominal value, where can be observed that these indices do not distinguish between increasing and reducing velocity. By comparing the $S F$ obtained, it is found a greater sensibility of the indices front to velocity variations than for amplitude changes.

Based on the experimental results and the above analysis, either $T^{2}$ or $Q$ Statistics can be nominated as a feature to track the stress condition in a material.

a)

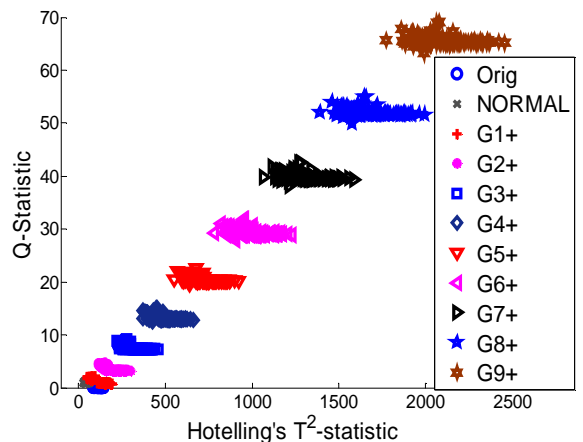

b)

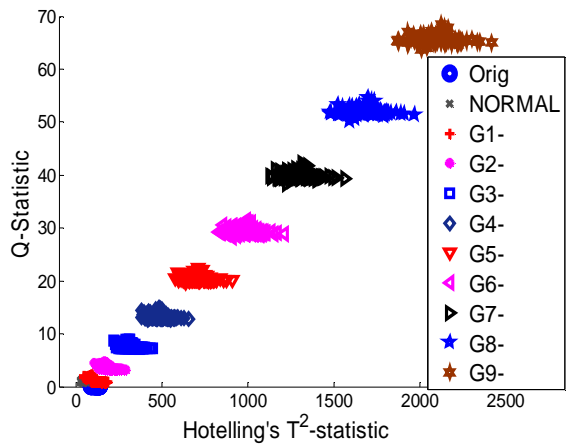

Figure 3: Q- $\mathrm{T}^{2}$ relationship for scaled versions of the nominal guided wave a) in steps of gains of $1 \%, b)$ in steps of attenuation of $1 \%$.

a)
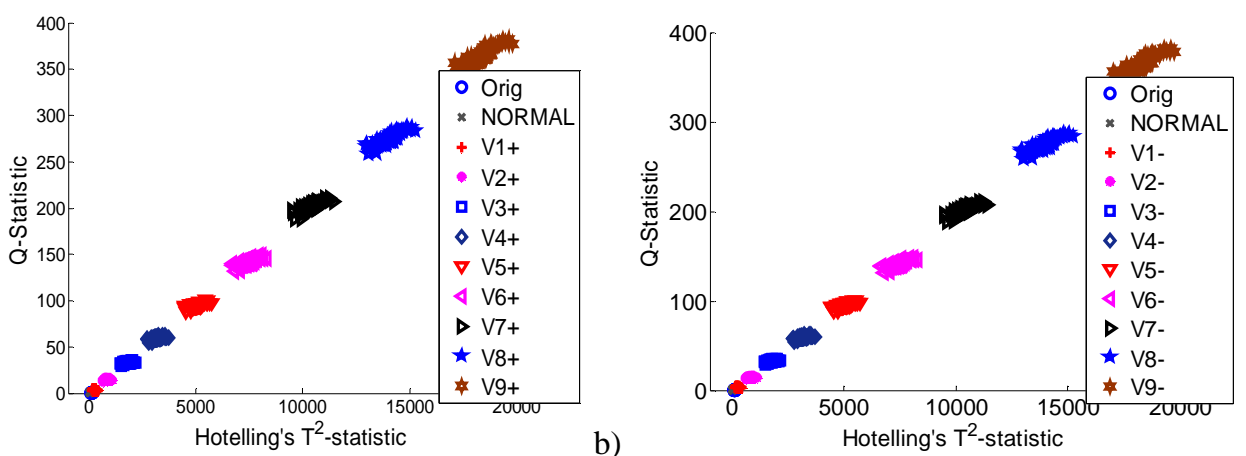

Figure 4: Q- $\mathrm{T}^{2}$ relationship for delayed versions of the nominal guided wave a) in steps of $0.4 \%$ of increasing (V1+ to V9+), b) in steps of $1 \%$ of decreasing (V1- to V9-).

\section{EXPERIMENTAL SETUP}

Experimental tests were performed on a 1" steel rod, where stress conditions are produced by a servohydraulic MTS universal machine mod 810 . The steel rod is excited with a 5 cycles Gaussian-modulated sinusoidal pulse via PZT. Both the guided wave and the PCA-based algorithm are programmed in Matlab, and a picoscope 2208 is used as DAQ system. The load magnitude is changed by steps of 
$22 \mathrm{kN}$, where each step belongs to a different stress scenario, denominated S1, S2, and so on consecutively until S9, which corresponds to a $90 \%$ of the yield stress.

A number of 100 experiments were performed and recorded for each stress scenario and every experiment is composed by 1000 samples for original signals and 2000 samples for the cross-correlated signals. The baseline model is obtained using only 70 experiments, while 30 are used to validate the model. The principal components were determined by using the baseline model, where a number of 60 were retained for a $99.8 \%$ of data variability.

In the diagnosis step, nine different scenarios $\mathrm{S} 1$ to $\mathrm{S} 9$ are projected onto the baseline model to calculate indices $T^{2}$ and Q-Statistics (see Figures 5). It can be observed that different stress scenarios can be distinguished by using the above statistical indices.

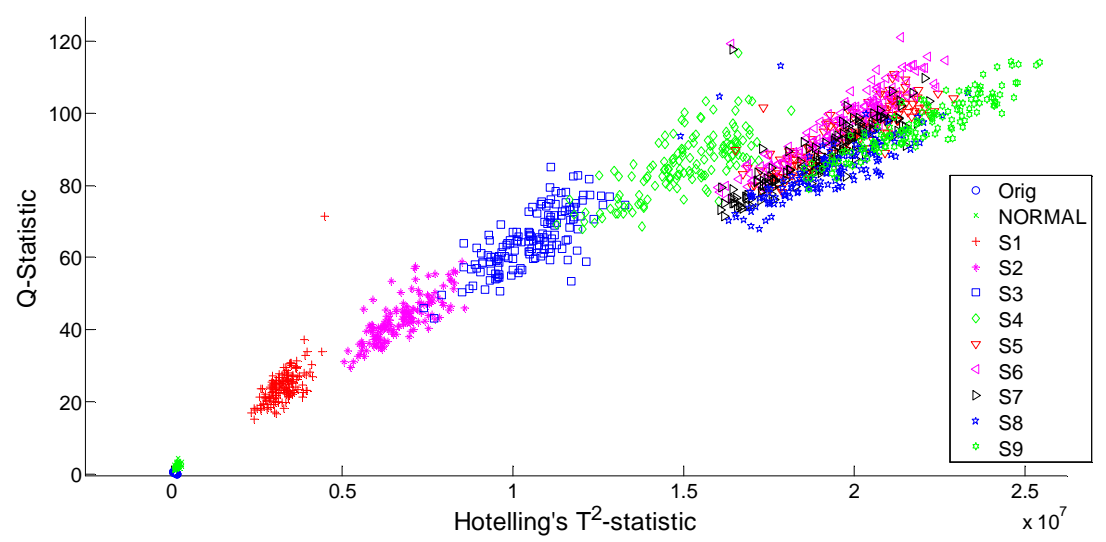

Figure 5: Indices Q-T² for different stress scenarios

\section{CONCLUSIONS}

In this paper a PCA based methodology for monitoring stress conditions in a steel rod is proposed and experimentally validated. It was verified the effectivity of the proposed monitoring scheme to yield stress features indicators and to discriminate different stress scenarios.

Experimental tests revealed changes in amplitude and phase velocity in the ultrasonic guided wave propagating in a medium under stress. These variations were exposed for the $T^{2}$ - $Q$ statistics indices after a PCA analysis was executed.

The studied scenarios covered most of the elastic region of the specimen: a 1 inch steel 1020 rod. In this region, the implemented algorithm was able to clearly distinguish different stress conditions via the couple coordinates $T^{2}-Q$.

Based on the capabilities of PCA to produce sensible indices to the stress a sensibility study is done using simulated signals. The results exposed a greater effect of the change of velocity in the values of the $T^{2}-Q$ than the variations in the amplitude.

In the $T^{2}-Q$ plane for the studied scenarios, it was observed a better definition of 
the cluster in the lower part of the elastic region of the material. So it, can be inferred the best capacities, associated to precision, for discriminating among the different stress scenarios for this region.

\section{REFERENCES}

1. Palanichamy P, Joseph A, Jayakumar T. 1995. "Ultrasonic velocity measurements for estimation of grain size in austenitic stainless steel," NDT \& International., 28:179-85.

2. Hakan G. C, I. Çam. 2007. "Comparison of magnetic Barkhausen noise and ultrasonic velocity measurements for microstructure evaluation of SAE 1040 and SAE 4140 steels". Materials Characterization., 58:447-454.

3. Allen, D. R., and Sayers, C. M.1984. "The measurement of residual stress in textures steel using an ultrasonics velocity combination technique.' Ultrasonics, 22: 179-188.

4. Stobbe David M. Master thesis. 2005. Acoustoelasticity in 7075-T651 Aluminum and Dependence of Third Order Elastic Constants on Fatigue Damage. School of Mechanical Engineering Georgia Institute of Technology.

5. Mohbacher H, E. Schneider , K. Goebbels. 1990. "Temperature dependence of third-order elastic constants." Presented at the $9^{\text {th }}$ International Conference on Experimental Mechanics,(3). Copenhagen; 1990. p. 1189-97.

6. Lhémery A, P. Calmon, S. Chatillon ,N. Gengembre. 2002. "Modeling of ultrasonic fields radiated by contact transducer in a component of irregular surface." Ultrasonics (40):231-236.

7. D. S. Hughes and J. L. Kelly. 1953. "Second-order elastic deformation of solids," Phys. Rev. (92):1145-1149.

8. Pao Y.-H., W. Sachse, and H. Fukuoka. 1984. "Acoustoelasticity and ultrasonic measurement of residual stress," Physical Acoustics, (17): 61-143.

9. Qu, J. and G. Liu. 1998. "Effects of residual stress on guided waves in layered media," Review of Progress in Quantitative Nondestructive Evaluation, (17): 1635-1642.

10. Stobbe David M. Master thesis. Acoustoelasticity in 7075-T651 Aluminum and Dependence of Third Order Elastic Constants on Fatigue Damage. School of Mechanical Engineering Georgia Institute of Technology, 2005.

11. Chaki, S., G. Bourse. 2009. "Guided ultrasonic waves for non-destructive monitoring of the stress levels in prestressed steel strands." Ultrasonics, (49):162-171.

12. Chaki, S., G. Bourse, 2009. "Stress Level Measurement in Prestressed Steel Strands Using Acoustoelastic Effect," Experimental Mechanics, (49): 673-681.

13. Gandhi, N. Determination of dispersion curves for acoustoelastic lamb wave propagation. MSc. Thesis. Georgia Institute of Technology. 2010.

14. Gharibnezhad, F, L. Mujica, J. Rodellar. 2015.“Applying Robust Variant of Principal Component Analysis as a Damage Detector in the Presence of Outliers". Mechanical System and Signal Processing, (50-51):467-479.

15. Huang B, K. Huang, H.S. Kim. 2014. "PCA-Based Damage Classification of Delaminated Smart Composite Structures using improved layerwise theory." Computer and Structures, (141): 26-35.

16. Mujica L., M. Ruiz, F. Pozo, J. Rodellar, J.Guemes, A. “A Structural Damage Detection Indicator Based on Principal Component Analysis and Statistical Hypothesis Testing". Smart Materials and Structures, (23-2),

17. Mujica L., J. Rodellar, A. Fernandez, J.Guemes, A. Q-statistic and T2-statistic PCA-based measures for damage assessment in structures. Structural Health Monitoring, (10): 539-553 